\title{
Respuesta al tratamiento periodontal de diabéticos tipo 2 con mal control metabólico y obesos intolerantes a la glucosa, con periodontitis severa
}

\author{
Impact of the periodontal therapy in both clinical and metabolic parameters in type 2 \\ diabetic and prediabetic obese patients with severe periodontitis
}

Pavez Correa V1, Araya Quintanilla A V², Baksai López N³

RESUMEN

Diabetes Mellitus (DM) es un factor de riesgo para periodontitis, a su vez, el control metabólico de los diabéticos se dificulta por la presencia de la infección periodontal, aumentando el riesgo de complicaciones crónicas y eventualmente, la mortalidad por diabetes.

Ambas enfermedades se relacionan porque los mediadores de inflamación generados en la infección periodontal y la translocación de bacterias de la cavidad oral a la circulación sistémica, podrían actuar como agravantes de la insulino resistencia y deteriorar el control glicémico.

Es evidente que el tratamiento oportuno de la periodontitis mejoraría la calidad de vida de los diabéticos; ayudándolos a alcanzar y mantener un adecuado control metabólico. El presente estudio evaluó el impacto de un protocolo de terapia periodontal en parámetros clínicos y metabólicos de pacientes DM2 y obesos prediabéticos, con el fin de contribuir a la inclusión de los diabéticos descompensados y prediabéticos en tratamientos periodontales exitosos, sin aumentar su riesgo médico.

Se seleccionó a 9 individuos con DM2 descompensados y 7 obesos intolerantes a la glucosa, todos con Periodontitis Crónica Generalizada Severa. Los pacientes fueron sometidos a tratamiento periodontal, sin modificar las indicaciones médicas, de acuerdo a un protocolo diseñado por nosotros. Los diabéticos mostraron una leve disminución en la $\mathrm{HbA} 1 \mathrm{c}$ a los 6 meses de realizado el tratamiento periodontal, no se registraron complicaciones, ni perdieron su condición de pacientes ambulatorios. La respuesta al tratamiento periodontal, fue similar en ambos grupos, con mejoría en todos los parámetros clínicos. Aunque los resultados obtenidos no son estadísticamente significativos, son muy promisorios.

Rev. Clin. Periodoncia Implantol. Rehabil. Oral Vol. 4(2); 50-53, 2011.

Palabras clave: Periodontitis, diabetes, obesidad, control metabólico.

\section{ABSTRACT}

Diabetes Mellitus (DM) is a risk factor for periodontitis, at the same time, the metabolic control of diabetic patients gets more difficult due to the presence of periodontal infection, increasing the risk of chronic complications and possibly, death by diabetes.

It is recognized that both diseases are related because the inflammation factors generated by the periodontitis as well as the oral cavity bacteria in the systemic circulation, may act as aggravating factor for insulin resistance and worsen the glycemic control.

It is clear that an opportune treatment of PD would improve the quality of life and the metabolic control of diabetic patients.

The objectives of the current paper are to evaluate the impact of the periodontal therapy in both clinical and metabolic parameters in type 2 diabetic and prediabetic obese patients and to demonstrate that uncontrolled diabetics can be treated successfully, without increasing their medical risk.

Nine DM2 and 7 glucose intolerant obese patients were selected. All of them suffering severe chronic periodontitis. All patients were treated following a protocol designed by us and medical indications were not modified.

The DM2 patients showed approximately a 1\% decrease in HbAlc after 6 months of the periodontal treatment. No complication was observed and no patient needed major medical assistance. The response to the periodontal treatment was similar in both groups, with a significant improvement in clinical parameters. Although the decrease in $\mathrm{HbA} 1 \mathrm{C}$ was not statistically significant, our results are very promising at the clinical setting.

Rev. Clin. Periodoncia Implantol. Rehabil. Oral Vol. 4(2); 50-53, 2011.

Key words: Periodontitis, diabetes, obesity, metabolic control.

\section{INTRODUCCIÓN}

La Diabetes Mellitus (DM) es un factor de riesgo para el desarrollo de periodontitis, incrementando su extensión y severidad ${ }^{(1-4)}$, y a su vez, el control metabólico de los pacientes diabéticos se hace más difícil por la presencia de la infección periodontal|(5-7), lo que aumenta el riesgo de complicaciones crónicas y eventualmente, la mortalidad por diabetes $^{(8)}$.

Ambas enfermedades se relacionan por los mediadores de inflamación generados en la infección periodontal: interleuquina 1 y 6 $(\mathrm{IL}-1,6)$ y factor de necrosis tumoral alfa (TNFa), principalmente, así como por la translocación de bacterias de la cavidad oral a la circulación sistémica. Estos mediadores inflamatorios y bacterias provocan una interferencia en la acción de los receptores de insulina, disminuyendo su sensibilidad ${ }^{(9-11)}$. Así, los diabéticos con periodontitis podrían tener elevados niveles séricos de TNFa, IL-6, proteína $C$ reactiva (PCR), leptina, molécula de adhesión intercelular 1 , soluble (sICAM-1) y menores niveles de adiponectina ${ }^{(12)}$. Todas estas moléculas podrían eventualmente actuar como agravantes de la insulino resistencia y deteriorar el control glicémico.

Además, existiría una asociación entre periodontitis severa y niveles altos de glicemia, en obesos no diabéticos. Saito et al. observaron que la pérdida de hueso alveolar se asocia con intolerancia a la glucosa en hombres japoneses sin diabetes ${ }^{(13)}$. Se sugiere que la periodontitis podría afectar el metabolismo de la glucosa en la población general, aunque en menor medida de lo que afecta a pacientes diabéticos ${ }^{(14)}$. La periodontitis se asocia a una ligera elevación de la hemoglobina glicosilada, y a su vez, a elevados niveles de glucosa sanguínea en adultos sin diabetes, lo que podría incrementar el riesgo de DM2 ${ }^{(13,14)}$.

Es evidente que el tratamiento oportuno de la periodontitis

1. Cirujano Dentista. Magíster en Ciencias Odontológicas Mención Periodontología. Universidad de Chile, Chile. Académico Universidad Mayor, Chile.

2. Médico Cirujano. Endocrinóloga. Universidad de Chile. Hospital Clínico Universidad de Chile, Chile.

3. Cirujano Dentista. Universidad de Chile, Chile.

Correspondencia autor: Violeta Pavez Correa. violetapavez@gmail.com. Trabajo recibido el 17/02/2011. Aprobado para su publicación el $27 / 04 / 2011$. Este trabajo forma parte del Proyecto Domeyko de Obesidad y Diabetes, de la Vicerrectoría de Investigación y Desarrollo de la Universidad de Chile: "Evaluación de marcadores bioquímicos y genéticos de daño endotelial y periodontal en individuos obesos normoglicemicos, intolerantes a la glucosa y diabéticos tipo 2 ". 
mejoraría la calidad de vida de los diabéticos; permitiendo alcanzar y mantener un adecuado control metabólico(15-21), con todas las implicancias médicas que eso conlleva ${ }^{(22,23)}$. Tradicionalmente, la atención odontológica del diabético se ha pospuesto hasta que el médico certifique que el paciente esté metabólicamente compensado; dando, a veces, alguna sugerencia respecto del uso de anestesia o antibioterapia. La ausencia de un protocolo de tratamiento periodontal para el diabético descompensado ha generado una discriminación negativa hacia estos pacientes, por la creencia que sus tratamientos serán poco exitosos y de alto riesgo médico-legal. Esta situación resulta especialmente paradójica si se sabe que la infección periodontal severa contribuye al deterioro metabólico de estos pacientes.

\section{TRATAMIENTO PERIODONTAL EN DIABÉTICOS}

Numerosos estudios de sujetos con DM2 y periodontitis han mostrado mejoría en el control metabólico(15-21) (disminución en 0.9 $-1 \%$ de la Hemoglobina glicosilada (HbA1c)) luego de ser sometidos a tratamiento periodontal convencional, adicionando o no antibióticos, probablemente debido a la disminución de IL-6, TNFa, PCR, leptina y el incremento de los niveles de adiponectina ${ }^{(18,20,21)}$. Esta forma tradicional de tratamiento se realiza por sextantes o cuadrantes de la boca, que se completa habitualmente en 4 a 6 semanas. Desde el año 1995, existe otro esquema de tratamiento llamado "desinfección completa de la boca", y se ejecuta en una o dos sesiones, dentro de $48 \mathrm{hrs}$. Este esquema se basa en la noción de que un grupo de dientes recientemente tratado podría ser recolonizado por bacterias patogénicas de los sacos periodontales de los dientes aún no tratados o de nichos extra-periodontales como amígdalas y lengua ${ }^{(24)}$. Hay que destacar que en los estudios de impacto de la terapia periodontal en diabéticos, en los que se ha evaluado la $\mathrm{HbA} 1 \mathrm{c}$ como parámetro de control metabólico, no se ha logrado el óptimo que es obtener un valor $<7 \%$, de acuerdo a los estándares definidos por la Asociación Americana de Diabetes (ADA) en el año 2009(25).

Nuestro objetivo fue evaluar el impacto sobre parámetros clínicos y metabólicos de pacientes DM2 y obesos prediabéticos, con Periodontitis crónica generalizada severa, de un protocolo de terapia periodontal basado en los principios de desinfección completa de la boca, pero incorporando la práctica y refuerzo del control de placa bacteriana por parte del paciente, con el fin de contribuir a la inclusión de los diabéticos descompensados y prediabéticos en tratamientos periodontales exitosos, sin aumentar su riesgo médico.

\section{MATERIAL Y MÉTODO}

Se seleccionó una muestra de 9 individuos con el diagnóstico de DM tipo 2 según los criterios de la ADA publicados en el año 2010(26): glicemia mayor de $126 \mathrm{mg} / \mathrm{dl}$ en ayunas o de $200 \mathrm{mg} / \mathrm{dl}$ o más, en cualquier momento del día, con $\mathrm{HbA} 1 \mathrm{c}$ mayor a $7 \%$ y 7 obesos intolerantes a la glucosa (IMC>30 y glicemias postcarga oral de $75 \mathrm{~g}$ de glucosa entre $141 \mathrm{y}$ $199 \mathrm{mg} / \mathrm{dl}$ ). La glicemia se determinó en suero por el método de la glucosa oxidasa. Para la HbA1c se utilizó una muestra de plasma y se determinó por un método fluorimétrico automatizado (Vitros 5.1 Johnson \& Johnson).

Todos los pacientes tenían más de 10 dientes funcionales, y en el periodontograma a lo menos 3 dientes de distinto sextante con a lo menos un sitio con NIC de $5 \mathrm{~mm}$ o más, en presencia de saco periodontal mayor a $4 \mathrm{~mm}$, en el mismo sitio; por lo cual su diagnóstico periodontal era Periodontitis Crónica Generalizada Severa. Se excluyeron del estudio los pacientes que consumían alguno de los siguientes medicamentos (o diagnóstico de las siguientes enfermedades): anticoagulantes, corticoides, antidepresivos, Imuran, Metotrexato, cardiopatías, depresión, enfermedad a la tiroides sin tratamiento, cáncer. El tamaño de la muestra, originalmente de 30 pacientes, resultó limitado por los estrictos criterios de inclusión y exclusión, así como por la falta de adherencia de algunos pacientes al protocolo de tratamiento y al seguimiento a los 6 meses.

Todos los pacientes firmaron una carta de consentimiento informado. El estudio fue aprobado por el Comité de Ética del Hospital Clínico y de la Facultad de Odontología de la Universidad de Chile y se basa en los principios de la declaración de Helsinki.

Los pacientes fueron sometidos a tratamiento periodontal, sin modificar las indicaciones médicas de cada individuo, de acuerdo al siguiente protocolo: $1^{\text {a }}$ Sesión: Instrucción de higiene oral, indicación de técnica de cepillado Charters, con cepillo suave y cepillos interproximales o seda dental, según el caso, y destartraje supragingival.

$2^{a}$ Sesión ( 1 semana después de la $1^{\text {a }}$ sesión): Destartraje subgingival y pulido radicular de hemiarcada superior e inferior derecha, e indicación de antibioterapia: Azitromicina $500 \mathrm{mg}$ cada $24 \mathrm{hrs}$ por 3 días (Ricilina ${ }^{\circledR}$ Laboratorios Recalcine). Refuerzo de higiene oral.

$3^{a}$ Sesión ( 2 días después de la $2^{a}$ sesión): Destartraje subgingival y pulido radicular de hemiarcada superior e inferior izquierda; refuerzo de higiene oral. Exodoncias si estuvieran indicadas.

$4^{\text {a }}$ Sesión ( 1 semana después de la $3^{\mathrm{a}}$ sesión): Control y refuerzo de higiene oral.

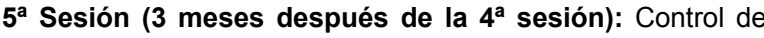
placa bacteriana y evaluación de signos de inflamación gingival.

$6^{a}$ Sesión ( 6 meses después de la $1^{\text {a }}$ sesión): Nuevo periodontograma. Los diabéticos fueron sometidos a un nuevo a examen de $\mathrm{HbA} 1 \mathrm{c}$.

La información clínica periodontal se analizó mediante diferencia de medias, en cada sujeto, antes y seis meses después del tratamiento periodontal. Los cambios en la PS y NIC después del tratamiento, se analizaron en subconjuntos de sitios, de acuerdo a la PS y NIC inicial: $\leq 3$ $\mathrm{mm}, 4$ a $6 \mathrm{~mm}$ y $\geq 7 \mathrm{~mm}$. Los dientes con indicación de exodoncia no fueron considerados en el análisis. La HbA1c se presenta como valor porcentual promedio $\pm D S$. Para analizar la diferencia antes y 6 meses después del tratamiento periodontal en el grupo de diabéticos, se utilizó la prueba de Wilcoxon. Se consideró significativo un valor de $p<0.05$.

\section{RESULTADOS}

En el examen periodontal inicial se observó una pobre condición periodontal, tanto en los sujetos DM2 descompensados (Tabla 1), como en los prediabéticos (Tabla 2). No habían recibido instrucción de higiene y no tenían el hábito de uso diario de seda dental y/o cepillos interproximales. Es importante hacer notar que estos pacientes presentaban un deterioro periodontal de tal magnitud que varios dientes ya estaban fuera del alcance terapéutico.

Tabla 1. Características periodontales basales de diabéticos tipo 2 descompensados. Se describe la condición periodontal inicial para cada uno de los nueve pacientes diabéticos a través de los parámetros: $\mathrm{NIC}=$ Nivel de inserción clínica, $\mathrm{P} 5=$ profundidad al sondaje $\mathrm{SS}=\%$ de sangramiento al sondaje, $\mathrm{PB}=$ Índice de placa bacteriana.

\begin{tabular}{|c|c|c|c|c|c|c|}
\hline \multirow{2}{*}{\begin{tabular}{|c} 
Pac \\
1
\end{tabular}} & \multicolumn{2}{|l|}{ NIC } & \multicolumn{2}{|l|}{ PS } & \multirow{2}{*}{$\frac{\text { SS }}{85 \%}$} & \multirow{2}{*}{$\frac{\text { PB }}{100 \%}$} \\
\hline & $\begin{array}{l}\leq 3 \mathrm{~mm} \\
4-6 \mathrm{~mm} \\
\geq 7 \mathrm{~mm}\end{array}$ & $\begin{array}{r}13.2 \% \\
63.2 \% \\
6.6 \% \\
\end{array}$ & $\begin{array}{l}\leq 3 \mathrm{~mm} \\
4-6 \mathrm{~mm} \\
\geq 7 \mathrm{~mm}\end{array}$ & $\begin{array}{r}82 \% \\
15 \% \\
1.8 \% \\
\end{array}$ & & \\
\hline 2 & $\begin{array}{l}\leq 3 \mathrm{~mm} \\
4-6 \mathrm{~mm} \\
\geq 7 \mathrm{~mm}\end{array}$ & $\begin{array}{r}66.0 \% \\
33.9 \% \\
0 \% \\
\end{array}$ & $\begin{array}{l}\leq 3 \mathrm{~mm} \\
4-6 \mathrm{~mm} \\
\geq 7 \mathrm{~mm}\end{array}$ & $\begin{array}{r}89.8 \% \\
10.1 \% \\
0 \% \\
\end{array}$ & $70 \%$ & $97 \%$ \\
\hline 3 & $\begin{array}{l}\leq 3 \mathrm{~mm} \\
4-6 \mathrm{~mm} \\
\geq 7 \mathrm{~mm}\end{array}$ & $\begin{array}{r}48.2 \% \\
45.3 \% \\
6.4 \% \\
\end{array}$ & $\begin{array}{l}\leq 3 \mathrm{~mm} \\
4-6 \mathrm{~mm} \\
\geq 7 \mathrm{~mm}\end{array}$ & $\begin{array}{r}77.5 \% \\
21 \% \\
1.4 \% \\
\end{array}$ & $68.1 \%$ & $93 \%$ \\
\hline 4 & $\begin{array}{l}\leq 3 \mathrm{~mm} \\
4-6 \mathrm{~mm} \\
\geq 7 \mathrm{~mm}\end{array}$ & $\begin{array}{l}29.6 \% \\
38.8 \% \\
31.4 \% \\
\end{array}$ & $\begin{array}{l}\leq 3 \mathrm{~mm} \\
4-6 \mathrm{~mm} \\
\geq 7 \mathrm{~mm}\end{array}$ & $\begin{array}{r}62.9 \% \\
33.3 \% \\
3.7 \% \\
\end{array}$ & $70 \%$ & $100 \%$ \\
\hline 5 & $\begin{array}{l}\leq 3 \mathrm{~mm} \\
4-6 \mathrm{~mm} \\
\geq 7 \mathrm{~mm}\end{array}$ & $\begin{array}{l}41.8 \% \\
47.0 \% \\
11.1 \% \\
\end{array}$ & $\begin{array}{l}\leq 3 \mathrm{~mm} \\
4-6 \mathrm{~mm} \\
\geq 7 \mathrm{~mm}\end{array}$ & $\begin{array}{r}82.6 \% \\
16.6 \% \\
0.6 \% \\
\end{array}$ & $73.6 \%$ & $100 \%$ \\
\hline 6 & $\begin{array}{l}\leq 3 \mathrm{~mm} \\
4-6 \mathrm{~mm} \\
\geq 7 \mathrm{~mm}\end{array}$ & $\begin{array}{r}9.2 \% \\
65.9 \% \\
24.7 \% \\
\end{array}$ & $\begin{array}{l}\leq 3 \mathrm{~mm} \\
4-6 \mathrm{~mm} \\
\geq 7 \mathrm{~mm}\end{array}$ & $\begin{array}{c}94.1 \% \\
5.9 \% \\
0 \% \\
\end{array}$ & $36.4 \%$ & $100 \%$ \\
\hline 7 & $\begin{array}{l}\leq 3 \mathrm{~mm} \\
4-6 \mathrm{~mm} \\
\geq 7 \mathrm{~mm}\end{array}$ & $\begin{array}{l}31.1 \% \\
53.3 \% \\
15.5 \% \\
\end{array}$ & $\begin{array}{l}\leq 3 \mathrm{~mm} \\
4-6 \mathrm{~mm} \\
\geq 7 \mathrm{~mm}\end{array}$ & $\begin{array}{c}86.6 \% \\
13.3 \% \\
0 \% \\
\end{array}$ & $50 \%$ & $95 \%$ \\
\hline 8 & $\begin{array}{l}\leq 3 \mathrm{~mm} \\
4-6 \mathrm{~mm} \\
\geq 7 \mathrm{~mm}\end{array}$ & $\begin{array}{l}23.5 \% \\
47.1 \% \\
29.2 \% \\
\end{array}$ & $\begin{array}{l}\leq 3 \mathrm{~mm} \\
4-6 \mathrm{~mm} \\
\geq 7 \mathrm{~mm}\end{array}$ & $\begin{array}{r}60.2 \% \\
35.2 \% \\
4.4 \% \\
\end{array}$ & $80.7 \%$ & $89 \%$ \\
\hline 9 & $\begin{array}{l}\leq 3 \mathrm{~mm} \\
4-6 \mathrm{~mm} \\
\geq 7 \mathrm{~mm}\end{array}$ & $\begin{array}{r}45.6 \% \\
49.6 \% \\
4.6 \%\end{array}$ & $\begin{array}{l}\leq 3 \mathrm{~mm} \\
4-6 \mathrm{~mm} \\
\geq 7 \mathrm{~mm}\end{array}$ & $\begin{array}{c}65.9 \% \\
30 \% \\
3.4 \%\end{array}$ & $63.8 \%$ & $100 \%$ \\
\hline
\end{tabular}


Tabla 2. Características periodontales basales de los pacientes prediabéticos. Se describe la condición periodontal inicial para cada uno de los siete pacientes pre diabéticos a través de los parámetros: $\mathrm{NIC}=$ Nivel de inserción clínica, $\mathrm{P} 5=$ profundidad al sondaje $\mathrm{SS}=\%$ de sangramiento al sondaje, $\mathrm{PB}=$ Índice de placa bacteriana.

\begin{tabular}{|c|c|c|c|c|c|c|}
\hline \multirow{2}{*}{$\begin{array}{c}\text { Pac } \\
1\end{array}$} & \multicolumn{2}{|l|}{ NIC } & \multicolumn{2}{|l|}{ PS } & \multirow{4}{*}{$\frac{\text { sS }}{46.7 \%}$} & \multirow{4}{*}{$\begin{array}{l}\text { PB } \\
98 \%\end{array}$} \\
\hline & $\leq 3 \mathrm{~mm}$ & $88 \%$ & $\leq 3 \mathrm{~mm}$ & $89.1 \%$ & & \\
\hline & $4-6 \mathrm{~mm}$ & $11.9 \%$ & $4-6 \mathrm{~mm}$ & $10.8 \%$ & & \\
\hline & $\geq 7 \mathrm{~mm}$ & $0 \%$ & $>7 \mathrm{~mm}$ & $0 \%$ & & \\
\hline \multirow[t]{3}{*}{2} & $\leq 3 \mathrm{~mm}$ & $31.9 \%$ & $\leq 3 \mathrm{~mm}$ & $62.5 \%$ & \multirow[t]{3}{*}{$60 \%$} & \multirow[t]{3}{*}{$92 \%$} \\
\hline & $4-6 \mathrm{~mm}$ & $61.3 \%$ & $4-6 \mathrm{~mm}$ & $36.6 \%$ & & \\
\hline & $\geq 7 \mathrm{~mm}$ & $5.8 \%$ & $\geq 7 \mathrm{~mm}$ & $0.8 \%$ & & \\
\hline \multirow[t]{3}{*}{3} & $\leq 3 \mathrm{~mm}$ & $30.2 \%$ & $\leq 3 \mathrm{~mm}$ & $-85.8 \%$ & \multirow[t]{3}{*}{$43.5 \%$} & \multirow[t]{3}{*}{$86 \%$} \\
\hline & $4-6 \mathrm{~mm}$ & $30.3 \%$ & $4-6 \mathrm{~mm}$ & $12.8 \%$ & & \\
\hline & $\geq 7 \mathrm{~mm}$ & $2.6 \%$ & $\geq 7 \mathrm{~mm}$ & $1.2 \%$ & & \\
\hline \multirow[t]{3}{*}{4} & $\leq 3 \mathrm{~mm}$ & $59.6 \%$ & $\leq 3 \mathrm{~mm}$ & $96 \%$ & \multirow[t]{3}{*}{$43.7 \%$} & \multirow[t]{3}{*}{$95 \%$} \\
\hline & $4-6 \mathrm{~mm}$ & $37.9 \%$ & $4-6 \mathrm{~mm}$ & $3.9 \%$ & & \\
\hline & $\geq 7 \mathrm{~mm}$ & $2.4 \%$ & $\geq 7 \mathrm{~mm}$ & $0 \%$ & & \\
\hline \multirow[t]{3}{*}{5} & $\leq 3 \mathrm{~mm}$ & $37 \%$ & $\leq 3 \mathrm{~mm}$ & $44.4 \%$ & \multirow[t]{3}{*}{$57.1 \%$} & \multirow[t]{3}{*}{$100 \%$} \\
\hline & $4-6 \mathrm{~mm}$ & $58.6 \%$ & $4-6 \mathrm{~mm}$ & $51.8 \%$ & & \\
\hline & $\geq 7 \mathrm{~mm}$ & $4.3 \%$ & $\geq 7 \mathrm{~mm}$ & $3.7 \%$ & & \\
\hline \multirow[t]{3}{*}{6} & $\leq 3 \mathrm{~mm}$ & $47.3 \%$ & $\leq 3 \mathrm{~mm}$ & $57.3 \%$ & \multirow[t]{3}{*}{$32 \%$} & \multirow[t]{3}{*}{$98 \%$} \\
\hline & $4-6 \mathrm{~mm}$ & $45.3 \%$ & $4-6 \mathrm{~mm}$ & $42 \%$ & & \\
\hline & $\geq 7 \mathrm{~mm}$ & $7.3 \%$ & $\geq 7 \mathrm{~mm}$ & $0.6 \%$ & & \\
\hline \multirow[t]{3}{*}{7} & $\leq 3 \mathrm{~mm}$ & $58.6 \%$ & $\leq 3 \mathrm{~mm}$ & $96 \%$ & \multirow[t]{3}{*}{$75 \%$} & \multirow[t]{3}{*}{$100 \%$} \\
\hline & $4-6 \mathrm{~mm}$ & $40.1 \%$ & $4-6 \mathrm{~mm}$ & $4 \%$ & & \\
\hline & $\geq 7 \mathrm{~mm}$ & $1.2 \%$ & $\geq 7 \mathrm{~mm}$ & $0 \%$ & & \\
\hline
\end{tabular}

El efecto sistémico de ésta intervención en pacientes DM2 descompensados mostró una disminución de $0.8 \%$ en la $\mathrm{HbA} 1 \mathrm{c}$ a los 6 meses de realizado el tratamiento periodontal (de $9.7 \pm 2.4 \%$ a $8.9 \pm 2.3 \%$ ), no alcanzando significancia estadística. Además, no se registraron complicaciones en los pacientes, ni perdieron su condición de pacientes ambulatorios (Figura 1).

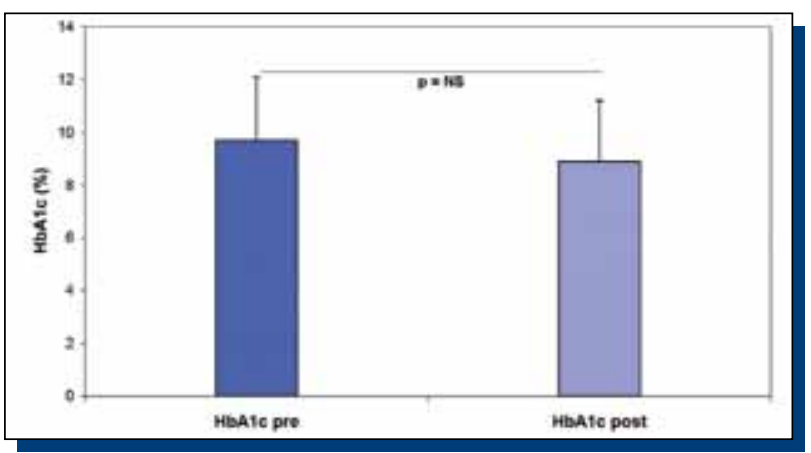

Figura 1. HbA1c antes y 6 meses post-tratamiento periodontal. Se muestra la variación en la hemoglobina glicosilada (HbA1c) antes y después de 6 meses del tratamiento periodontal. Observamos una importante disminución de dicha proteína aunque sin alcanzar significancia estadística.

La respuesta clínica al protocolo de tratamiento periodontal presentado en este trabajo fue muy buena, con valores similares en ambos grupos de sujetos. A los 6 meses, se observó una significativa mejoría en los niveles de inserción clínica (Tabla 3) y en la profundidad al sondaje (Tabla 4). En ambas mediciones, los resultados de los pacientes diabéticos fueron mejores que aquellos de los prediabéticos.

Tabla 3. Cambios en el N IC de los pacientes a los 6 meses del tratamiento. Se presenta la variación en el Nivel de inserción clínica (NIC) en ambos grupos de pacientes, seis meses después del tratamiento periodontal. Observamos que los pacientes diabéticos mostraron una ganancia de inserción mayor que los pacientes pre diabéticos, en particular en los sitios que tenían un NIC inicial mayor o igual a $7 \mathrm{~mm}$.

\begin{tabular}{|l|l|l|c|c|}
\hline & \multicolumn{2}{|c|}{ Diabéticos } & \multicolumn{2}{c|}{ Prediabéticos } \\
\hline NIC inicial & NIC final & Variación & NIC final & Variación \\
\hline$\$ 3 \mathrm{~mm}$ & $2.5 \mathrm{~mm}$ & $0.07 \mathrm{~mm}$ & $1.6 \mathrm{~mm}$ & $-0.1 \mathrm{~mm}$ \\
\hline $\mathbf{4 6 \mathrm { mm }}$ & $3.72 \mathrm{~mm}$ & $1.02 \mathrm{~mm}$ & $3.61 \mathrm{~mm}$ & $1 \mathrm{~mm}$ \\
\hline$\geq 7 \mathrm{~mm}$ & $5.3 \mathrm{~mm}$ & $2.40 \mathrm{~mm}$ & $5 \mathrm{~mm}$ & $2.2 \mathrm{~mm}$ \\
\hline
\end{tabular}

Tabla 4. Cambios en la PS de los pacientes a los 6 meses del tratamiento. Se presenta la variación en la Profundidad de Sondaje (PS) en ambos grupos de pacientes, seis meses después del tratamiento periodontal. Al igual que lo observado respecto al NIC, los pacientes diabéticos mostraron una mayor disminución en la profundidad de los sacos que los pacientes prediabéticos, en particular en los sitios que se situaban en el tramo de PS inicial mayor o igual a $7 \mathrm{~mm}$.

\begin{tabular}{|lll|l|l|}
\hline \hline & \multicolumn{2}{c|}{ Diabéticos } & \multicolumn{2}{c|}{ Pre diabéticos } \\
\hline PS inicial & PS final & Variación & PS final & Variación \\
\hline $\mathbf{3 \mathbf { m m }}$ & $2.0 \mathrm{~mm}$ & $0.31 \mathrm{~mm}$ & $2.0 \mathrm{~mm}$ & $0.2 \mathrm{~mm}$ \\
\hline $\mathbf{4 6} \mathrm{mm}$ & $2.6 \mathrm{~mm}$ & $1.91 \mathrm{~mm}$ & $2.7 \mathrm{~mm}$ & $1.91 \mathrm{~mm}$ \\
\hline $\mathbf{7} \mathrm{mm}$ & $3.4 \mathrm{~mm}$ & $4.04 \mathrm{~mm}$ & $3.4 \mathrm{~mm}$ & $3.7 \mathrm{~mm}$ \\
\hline
\end{tabular}

Los sitios residuales con PS $\geq 4 \mathrm{~mm}$ a los 6 meses de realizado el tratamiento, fue de $2.9 \%$, (Figura 2), corresponden principalmente a sitios con profundidades iniciales $\geq 7 \mathrm{~mm}$ y dichos sitios deberán ser tratados más allá de los límites del protocolo de tratamiento periodontal propuesto, mediante un colgajo de acceso periodontal, en la medida que el endocrinólogo lo autorice. La terapia de soporte periodontal, en todos los casos, se programó cada 2 meses.

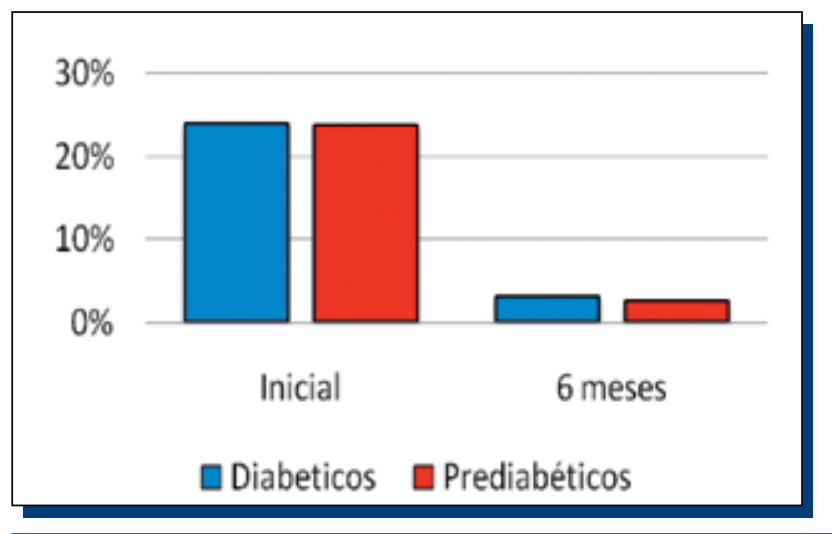

Figura 2. Porcentaje de sitios con PS $\geq 4 \mathrm{~mm}$. Se muestra la variación porcentual de los sitios con Profundidad de Sondaje mayor o igual a $4 \mathrm{~mm}$. La figura muestra que el porcentaje de dichos sitios disminuyó considerablemente después de 6 meses del tratamiento, tanto para pacientes DM2 como para pacientes prediabéticos.

\section{DISCUSIÓN}

El presente estudio evaluó el impacto de un protocolo de tratamiento periodontal, propuesto por nosotros, en parámetros clínicos y metabólicos de pacientes DM2 y obesos prediabéticos.

El examen inicial de estos pacientes mostró una condición periodontal deplorable. Ningún sujeto había recibido instrucciones de higiene ni tratamiento periodontal, lo que resulta impresentable siendo pacientes de riesgo médico, portadores de una infección crónica no tratada. Es importante hacer notar que muchos de ellos presentaban un deterioro periodontal de tal magnitud que varios dientes ya estaban fuera del alcance terapéutico.

La respuesta clínica al tratamiento periodontal, fue similar en ambos grupos de sujetos. A los 6 meses, se observó mejoría en todos los parámetros clínicos; los sitios residuales con PS $\geq 4 \mathrm{~mm}$ a los 6 meses de realizado el tratamiento, no alcanzaron al $3 \%$ de los sitios. Nuestros resultados en diabéticos descompensados concuerdan con los obtenidos en otro estudio, en diabéticos compensados, donde se comparó el tratamiento no quirúrgico convencional con el tratamiento periodontal de desinfección de boca completa; obteniéndose mejorías similares en los parámetros periodontales, después de 3 y 6 meses $^{(27)}$. Algunos autores señalan que los sujetos con buen control metabólico exhibieron mejores resultados en el NIC después de 6 meses, comparado con los sujetos con mal control metabólico ${ }^{(21)}$, mientras otros estudios muestran resultados similares. Pocos estudios cuentan con la participación de médicos endocrinólogos en la determinación del estado metabólico de los diabéticos, previo a la terapia periodontal, por lo cual podría haber algunos casos en que su categorización fuera motivo de controversia.

Estudios recientes, muestran que el tratamiento periodontal no quirúrgico intensivo (6 horas de destartraje y pulido radicular con un instrumento ultrasónico) produce aumento significativo de la respuesta 
inflamatoria sistémica en sujetos con periodontitis y buena salud general. Este aumento de la inflamación sistémica podría inducir una descompensación metabólica en pacientes con control adecuado de su diabetes y aumentar la descompensación en pacientes con diabetes no compensada ${ }^{(28)}$. Por esta razón, nos parece aconsejable que el tratamiento periodontal en diabéticos se efectúe en forma paulatina y sesiones cortas, reduciendo al mínimo la inflamación gingival, enfatizando el control personal de placa bacteriana, antes de efectuar acciones invasivas como el pulido radicular y eventualmente, cirugía periodontal.

La respuesta aumentada a endotoxemia y bacteremia en los diabéticos, las alteraciones en la cicatrización y reparación tisular y la posibilidad de aumento del riesgo cardiovascular en estos pacientes, fundamentan el uso de antibióticos como coadyudante del tratamiento periodontal(29).

Si bien habría sido interesante incorporar los resultados de $\mathrm{HbA} 1 \mathrm{c}$ a los 3 meses de realizado el tratamiento periodontal, para observar la evolución de ese valor en el tiempo, preferimos hacerlo a los 6 meses post-tratamiento para detectar cambios que podríamos considerar más estables, a mediano plazo.

El efecto de esta intervención en los pacientes DM2 descompensados fue una disminución en la $\mathrm{HbA} 1 \mathrm{c}$, no estadísticamente significativa, a los 6 meses de realizado el tratamiento periodontal pero, hay que recalcar que no hubo cambios en la terapia hipoglicemiante en ninguno de estos pacientes. Si consideramos que algunas de las terapias orales disponibles para el tratamiento de la diabetes 2 tampoco logran disminuir la HbA1c en más $0.5-1 \%$, podríamos decir que el tratamiento periodontal tiene un efecto significativo desde el punto de vista clínico, en el control metabólico de algunos diabéticos ${ }^{(25,30)}$.

En base a los presentes resultados, se concluye que el protocolo de tratamiento periodontal propuesto en este estudio es simple y efectivo en estos pacientes de riesgo médico, sin alterar su condición sistémica. Además, al no registrarse complicaciones en los pacientes, es posible pensar que en un futuro cercano los diabéticos podrán acceder a tratamiento odontológico oportuno y no seguirán siendo discriminados debido a su condición sistémica o premedicados sin un fundamento médico que sustente tal medida.

Aunque los resultados obtenidos no son estadísticamente significativos, son muy promisorios. Esta primera experiencia aplicando este protocolo de tratamiento a sólo 16 personas, hace necesario continuar trabajando con un mayor número de sujetos.

\section{CONFLICTOS DE INTERÉS} ningún tipo.

\section{REFERENCIAS BIBLIOGRÁFICAS}

1. Karjalainen K, Knuuttila M, von Dickholff K. Association of severity of periodontal disease with organ complications in type 1 diabetic patients. J Periontal, 1994; 65: 1067-1072.

2. Novak MJ, Potter RM, Blodgett J, Ebersole JL. Periodontal disease in Hispanic Americans with type 2 diabetes. J Periodontol, 2008; 79: 629636.

3. Nunn ME. Understanding the etiology of periodontitis: An overview of periodontal risk factors. Periodontol 2000, 2003; 32: 11-23.

4. Wang T-T, Chen TH-H, Wang P-E, Lai H, Lo M-T, Chen PY-C, Chiu SY$\mathrm{H}$. A population-based study on the association between type 2 diabetes and periodontal disease in 12, 123 middle-aged Taiwanese (KCIS No. 21). J Clin Periodontol, 2009; 36: 372-379.

5. Taylor GW. Bidirectional interrelationships between diabetes and periodontal diseases: An epidemiologic perspective. Ann Periodontol, 2001; 6: 99-112.

6. Grossi SG, Genco RJ. Periodontal disease and diabetes mellitus: A two-way relationship. Ann Periodontol, 1998; 3: 51-61.

7. Pavez V, Araya V, Rubio A, Ríos L, Meza P, Martínez B. Estado de salud periodontal en diabéticos tipo 1 de 18 a 30 años de edad, de Santiago de Chile. Rev Med de Chile, 2002; 130: 402-408.

8. Tharstensson $\mathrm{H}$, Kuylenslorna J, Hugoson. Medical status and complications in relation on to periodontal disease experience in insulindependent diabetics. J Clin Periodontol, 1996; 23: 194-202.

9. Genco RJ, Grossi SG, Ho A, Nishimura F, Murayama Y. A proposed model linking inflammation to obesity, diabetes, and periodontal infections. J Periodontol, 2005; 76: 2075-2084.

10. Kanoty H, Foinstein R, Papa MZ, et al. Tumor necrosis factor alphainduced phosphorylation of insulin receptor substrate (IRS-1). Possible mechanism of suppression of insulin-stimulated tyrosine phosphorylation of IRS-1. J Biol Chem, 1995; 270: 23780-23784.

11. Mealey BL, Ocampo GL. Diabetes mellitus and periodontal disease. Periodontol 2000, 2007; 44: 127-153.

12. Kardeşler L, Buduneli N, Çetinkalp S, Denis F, Kinane. Adipokines and inflammatory mediators following initial periodontal treatment in type 2 diabetic chronic periodontitis patients. Journal of Periodontology, Copyright 2009 DOI: $10.1902 /$ jop.2009.090267.

13. Saito T, Shimazaki Y, Koga T, Tsuzuki M, Ohshima A. Relationship between upper body obesity and periodontitis. J Dent Res, 2001; 80: 1631-1636.

14. Ryan EW, Larry FW, Bryan SM. A pilot study of glycosylated hemoglobin levels in priodontitis cases and healthy controls. J Periodontol, July 2009; 80(7): 1057-1061.

15. Taylor GW. The effects of periodontal treatment on diabetes. $J A m$ Dent Assoc, 2003; 134: 41S-48S.

16. Faria-Almeida R, Navarro A, Bascones A. Clinical and metabolic changes after conventional treatment of type 2 diabetic patients with chronic periodontitis. J Periodontol, Apr 2006; 77(4): 591-598.
17. Rodrígues DC, Taba MJ, Novaes AB, Souza SL, Grisi MF. Effect of non surgical periodontal therapy on glycemic control in patients with type 2 diabetes mellitus. J Periodontol, 2003; 74: 1361-1367.

18. O'Connell PA, Taba M, Nomizo A et al. Effects of periodontal therapy on glycemic control and inflammatory markers. J Periodontol, 2008; 79: 774-783.

19. Kiran M, Arpak N, Ünsal E, Erdoðan MF. The effect of improved periodontal health on metabolic control in type 2 diabetes mellitus. $J$ Clin Periodontol, 2005; 32: 266-272.

20. Navarro-Sánchez AB, Faria-Almeida R, Bascones-Martinez A. Effect of non-surgical periodontal therapy on clinical and immunological response and glycemic control in type 2 diabetic patients with moderate periodontitis. J Clin Periodontol, 2007; 34: 835-843.

21. da Cruz GA, de Toledo S, Sallum EA et al. Clinical and laboratory evaluations of non-surgical periodontal treatment in subjects with diabetes mellitus. J Periodontol, 2008; 79: 1150-1157.

22. Nesse W, Linde A, Abbas F, Spijkervet FKL, Dijkstra PU, de Brabander EC, Gerstenbluth I, Vissink A. Dose-response relationship between periodontal inflamed surface area and $\mathrm{HbA} 1 \mathrm{c}$ in type 2 diabetics. $J$ Clin Periodontol, 2009; 36: 295-300.

23. Stratton IM, Adler Al, Neil HAW et al. Association of glycemia with macrovascular and microvascular complications of type 2 diabetes (UKPDS 35): prospective observational study. Bri Med J, 2000; 321: 405-412.

24. Cosí G, Corbet EF, Ishikawa I. A full-mouth disinfection approach to nonsurgical periodontal therapy-prevention of reinfection from bacterial reservoirs. Periodontology 2000, 2004; 36: 166-178.

25. Nathan D, Buse J, Davidson M, Ferrannini E, Holman R, Sherwin R, Zinman B. Medical management of hyperglycemia in type 2 diabetes: A consensus algorithm for the initiation and adjustment of therapy. A consensus statement of the American Diabetes Association and the European Association for the Study of Diabetes. Diabetes Care, 2009; 32: 193-203.

26. American Diabetes Association. Diagnosis and Classification of Diabetes Mellitus. Diabetes Care, 2010; 33 (Supplement 1): S62-S69.

27. Santos V, Lima J, De Mendoncxa A, Máximo M, Faveri M, Duarte P. Effectiveness of full-mouth and partial-mouth scaling and root planing in treating chronic periodontitis in subjects with type 2 diabetes. $J$ Periodontol, August 2009; 80(8): 1237- 1245.

28. Peng Li, Lu He, Yue-qin Sha, Qing-xian Luan. Relationship of metabolic syndrome to chronic periodontitis. J Periodontol, April 2009; 80(4): 541-549.

29. Grossi S, López N. Tratamiento odontológico en el paciente diabético. Guía de tratamiento odontológico para pacientes diabéticos. Odontología Basada en Evidencias, 2008; 1(2): 8-21.

30. Nathan DM. Finding new treatments for diabetes-how many, how fast...how good? N Engl J Med, 2007; 56: 437-440. 